\title{
EMPREGO DA TÉCNICA DE CORRENTES PARASITAS PARA A INSPEÇÃO DE REVESTIMENTOS DE TANQUES DE ARMAZEMENTO *
}

\author{
Marcella Grosso Lima' \\ Clara Johanna Pacheco ${ }^{2}$ \\ Andre Henrique Mascarenhas Lima ${ }^{3}$ \\ Mónica Patricia Arenas Correa 4 \\ Isabel Cristina Pereira Magarit-Mattos ${ }^{5}$ \\ Sergio Damasceno Soares ${ }^{6}$ \\ Gabriela Ribeiro Pereira ${ }^{7}$
}

\section{Resumo}

O objetivo deste trabalho é pesquisar sobre as principais falhas apresentadas por revestimentos anticorrosivos modernos comumente empregados para proteção interna de tanques de armazenamento de produtos petroquímicos e avaliar a capacidade da técnica de correntes parasitas para a inspeção não destrutiva deste material. Nesse contexto, as vantagens e limitações da técnica são analisadas. A aquisição de dados foi realizada considerando as propriedades isolantes dos revestimentos nas condições como aplicado e também após o envelhecimento. Amostras foram preparadas com defeitos controlados inseridos no substrato de aço carbono a fim de simular defeitos de corrosão localizada sob o revestimento. A detecção dos defeitos foi avaliada nas amostras com diferentes espessuras e também com a presença de óxido de ferro no interior dos defeitos a fim de simular áreas de corrosão localizada. Através da comparação entre os resultados obtidos no C-Scan original e após o processamento de imagem, foi possível observar uma melhoria significativa na eliminação dos falsos defeitos relacionados a variação da espessura inerente do revestimento pela limiarização multilevel. Também foi possível através do efeito do lift-off distinguir os defeitos reais e falsos defeitos. Nesse aspecto, a técnica de correntes parasitas se mostrou capaz de detectar defeitos neste tipo de material.

Palavras-chave: Correntes Parasitas; Revestimentos; Corrosão.

\section{EDDY CURRENT AND INSPECTION OF COATINGS FOR STORAGE TANKS Abstract}

The aim is to make a survey of typical flaws presented by modern anticorrosive coatings typically employed for in-side protection of petrochemicals storage tanks and discuss what can be expected from nondestructive inspection with conventional eddy current technique (ECT). In this sense, the advantages and limitations of the method are discussed. Conditions for data acquisition considered the insulating properties of as-applied coatings and after aging. Samples were prepared with controlled defects machined on plain carbon steel, in order to simulate undercoating localized corrosion. Detectability was evaluated for as-applied insulating coatings with different thicknesses and also the presence of iron oxide inside the simulated corrosion spots were considered. Through the comparison between the results obtained in the original CScan and after the image processing, it could be concluded that the multilevel thresholding generated a significant improvement in the elimination of false defects related to the inherent variation of thickness of the coating. It was also possible through the lift-off effect to distinguish between real defects and false defects. In this respect, the eddy current testing has proved to be capable of detecting defects in this type of material.

Keywords: Eddy Current Testing; Coatings; Corrosion 
1 Engenheira Metalurgista, M. Sc, Pesquisadora, Laboratório de Ensaios Não Destrutivos, Corrosão e Soldagem (LNDC)/COPPE/UFRJ.

2 Física, D. Sc, Pesquisadora, Laboratório de Ensaios Não Destrutivos, Corrosão e Soldagem (LNDC)/COPPE/UFRJ..

3 Engenheiro de Materiais, Eng., Laboratório de Ensaios Não Destrutivos, Corrosão e Soldagem (LNDC)/COPPE/UFRJ.

4 Engenheira Física, M. Sc, Doutoranda, Laboratório de Ensaios Não Destrutivos, Corrosão e Soldagem (LNDC)/COPPE/UFRJ.

5 Engenheira Química, D. Sc, Professora Adjunta, Departamento de Engenharia Metalúrgica e de Materiais/ Universidade Federal do Rio de Janeiro (UFRJ)/COPPE.

6 Engenheiro Mecânico, D. Sc, CENPES/PETROBRAS

7 Física, D. Sc, Professora Adjunta, Departamento de Engenharia Metalúrgica e de Materiais/ Universidade Federal do Rio de Janeiro (UFRJ)/COPPE. 


\section{INTRODUÇÃO}

Os revestimentos têm sido amplamente aplicados para a proteção de substratos metálicos contra a corrosão [1]. Ao longo das últimas décadas, o emprego de revestimentos compósitos anticorrosivos tem sido considerado uma opção atraente para as instalações industriais que operam em condições ambientais severas, como altas temperaturas, meios ácidos e tensão mecânica por diferentes agentes. A inspeção destes revestimentos torna-se necessária devido a presença de empolamento, falha de aderência e corrosão abaixo do revestimento. Essas falhas podem ocorrer tanto como consequência de um procedimento de aplicação inadequado como também pela inerência ao processo de envelhecimento dos revestimentos. Neste contexto, o interesse na utilização de técnicas não destrutivas é justificado pela subjetividade conferida pela inspeção visual; dificuldade no acesso de toda a estrutura e na detecção de falhas entre as camadas e sob o revestimento.

Dentre as técnicas não destrutivas, neste trabalho foi avaliada a técnica de correntes parasitas convencional. Esta técnica, operando com uma única frequência senoidal, apesar de ser amplamente utilizada em diversas aplicações industriais [2], apresenta limitações referentes a profundidade de penetração quando aplicada em materiais não condutores [3]. Entretanto, considerando o método de lift-off [4], resultados significativos têm sido reportados [3,5-7].

O objetivo deste trabalho é analisar a performance da técnica de correntes parasitas na detecção de corrosão localizada sob a camada de revestimentos compósitos que são utilizados em tanques de armazenamento de petroquímicos. Além disso, também se pretende neste trabalho destacar a importância do conhecimento prévio das condições do revestimento para assegurar uma confiabilidade maior nos resultados obtidos em plantas industriais.

\section{MATERIAIS E MÉTODOS}

Para este trabalho foram avaliadas três amostras (A1, A2 e A3). Cada amostra é constituída de um substrato de aço carbono na forma de uma chapa de dimensões $100 \mathrm{~mm} \times 150 \mathrm{~mm} \times 4.7 \mathrm{~mm}$. Previamente a inserção dos defeitos, as superfícies dos substratos foram tratadas com um jateamento abrasivo e desengraxadas com tolueno a fim de retirar qualquer resíduo. O perfil de rugosidade obtido nas superfícies dos substratos foi de aproximadamente $70 \mu \mathrm{m}$.

Após a etapa de preparação superficial e anteriormente a aplicação dos revestimentos, foram usinados furos de diferentes profundidades e diâmetros nos substratos a fim de simular defeitos de perda de espessura no substrato devido a corrosão localizada. Em alguns defeitos, houve a inserção de óxido de ferro $\left(\mathrm{Fe}_{3} \mathrm{O}_{4}\right)$ nos furos para simular a presença de produto de corrosão abaixo da camada de revestimento. Na tabela 1 são apresentadas as especificações dos defeitos inseridos em cada amostra. 
Tabela 1. Especificações dos defeitos nas amostras $A 1, A 2$ e $A 3$

\begin{tabular}{ccccccc}
\hline Amostras & \multicolumn{2}{c}{ A1 } & \multicolumn{2}{c}{ A2 } & \multicolumn{2}{c}{ A3 } \\
\hline $\begin{array}{c}\text { Presença } \\
\text { de } \mathbf{F e}_{3} \mathbf{O}_{4}\end{array}$ & \multicolumn{2}{c}{ Não } & \multicolumn{2}{c}{ Não } & \multicolumn{2}{c}{ Sim } \\
\hline Defeito & $\begin{array}{c}\text { Diâmetro } \\
(\mathrm{mm})\end{array}$ & $\begin{array}{c}\text { Profundidade } \\
(\mathrm{mm})\end{array}$ & $\begin{array}{c}\text { Diâmetro } \\
(\mathrm{mm})\end{array}$ & $\begin{array}{c}\text { Profundidade } \\
(\mathrm{mm})\end{array}$ & $\begin{array}{c}\text { Diâmetro } \\
(\mathrm{mm})\end{array}$ & $\begin{array}{c}\text { Profundidade } \\
(\mathrm{mm})\end{array}$ \\
\hline 1 & 5.45 & 1.50 & 5.41 & 1.00 & 5.65 & 3.43 \\
\hline 2 & 5.25 & 2.30 & 5.36 & 1.90 & 5.56 & 2.54 \\
\hline 3 & 5.25 & 2.80 & 5.26 & 2.37 & 5.53 & 1.47 \\
\hline 4 & 10.75 & 1.50 & 11.20 & 1.53 & - & - \\
\hline 5 & 11.00 & 2.15 & 11.20 & 1.93 & - & - \\
\hline 6 & 10.84 & 2.70 & 11.24 & 3.00 & - & - \\
\hline
\end{tabular}

Após a inserção dos defeitos, a aplicação do revestimento foi realizada em duas etapas. Na primeira etapa foi aplicada uma fina camada do revestimento ainda no estado líquido sobre o substrato, contornando a áreas dos defeitos. Na segunda etapa, antes da cura da fina camada de revestimento, é adicionada por cima desta uma película (filme) do próprio revestimento já curado, desta forma a camada ainda não curada do revestimento desempenha a função de um adesivo. Para este trabalho, foram escolhidos dois tipos de revestimentos compósitos anticorrosivos que são comercialmente aplicados em tanques de armazenamento. Na Tabela 2 são apresentadas as principais características dos revestimentos e também os parâmetros de cada amostra.

Tabela 2. Informações técnicas dos revestimentos e descrição das amostras

\begin{tabular}{|c|c|c|c|}
\hline $\begin{array}{c}\text { Composição do } \\
\text { Revestimento }\end{array}$ & Indicação de uso & Amostra & $\begin{array}{c}\text { Espessura do } \\
\text { revestimento } \\
(\mu \mathrm{m})\end{array}$ \\
\hline \multirow{2}{*}{$\begin{array}{c}\text { Resina epóxi } \\
\text { multifuncionais com carga } \\
\text { de flocos de vidro. } 98 \% \\
\text { sólidos. Agente de cura: } \\
\text { poliamida modificada. Cura } \\
\text { total: } 7 \text { dias em } 25^{\circ} \text { C. }\end{array}$} & \multirow{2}{*}{$\begin{array}{l}\text { Resistente à produtos } \\
\text { químicos agressivos, } \\
\text { incluindo solventes e } \\
\text { ácidos fortes à } \\
\text { temperatura ambiente } \\
\text { até } 80^{\circ} \mathrm{C} \text {. }\end{array}$} & $\mathrm{A} 1$ & $972 \pm 55$ \\
\hline & & A3 & $582 \pm 125$ \\
\hline $\begin{array}{c}\text { Resina epóxi } \\
\text { multifuncionais com reforço } \\
\text { de mistura de minerais. } \\
\text { 100\% sólidos. Agente de } \\
\text { cura: aminocicloalifático } \\
\text { modificado. Cura total: } 250 \\
\text { horas em } 25^{\circ} \mathrm{C} \text {. }\end{array}$ & $\begin{array}{l}\text { Resistente à acetona, } \\
\text { soluções alcalinas, } \\
\text { diesel, etanol, } \\
\text { gasolina, álcool, } \\
\text { querosene, metanol, } \\
\text { nafta, água salgada, } \\
\text { tolueno, ácido acético } \\
\text { (5\%), ácido nítrico } \\
\text { (10-60\%) e ácido } \\
\text { sulfúrico (30-98\%). }\end{array}$ & $\mathrm{A} 2$ & $376 \pm 60$ \\
\hline
\end{tabular}

As inspeções pela técnica de correntes parasitas foram realizadas utilizando um sistema comercial de correntes parasitas (ZETEC) e com a sonda na faixa de frequência entre $5 \mathrm{kHz}$ e $50-\mathrm{kHz}$. Para a aquisição dos dados resultantes das inspeções foi realizada por um osciloscópio modelo Tektronix MSO 4034, associado a um software desenvolvido em linguagem Labview para a aquisição do sinal, análise dos dados e controle da mesa de varredura nas posições $X Y Z$. A aquisição dos sinais foi realizada através da movimentação da sonda, com auxílio da mesa de varredura, ao longo da superfície de cada amostra nas direções $x$ e y. A resolução adotada para 
a varredura foi de $1 \mathrm{~mm}$ por passo. O equipamento de correntes parasitas foi configurado com a frequência de $10 \mathrm{kHz}$, ganho de $33 \mathrm{~dB}$, ângulo de fase igual a 122은 e corrente de 0,02 mA enviada a sonda. A calibração prévia a inspeção foi realizada numa região sem defeitos, próxima a borda de cada amostra e tal região foi definida como o ponto $(0,0)$ no sistema de coordenadas $X Y$ da mesa de varredura.

A partir do plano de impedância gerado pelos sinais obtidos nas inspeções [8], a variação do ângulo de fase e da amplitude são os parâmetros mais importantes para a análise. Neste estudo, foi considerado apenas a amplitude como um parâmetro de análise. Esses dados de amplitude obtidos da varredura foram armazenados na forma de matrizes, possibilitando a construção de mapas C-Scan para cada amostra avaliada.

Além da análise dos resultados obtidos pela inspeção por correntes parasitas em cada amostra, também optou-se nesse trabalho em avaliar a metodologia de processamento de imagem a ser aplicada nas imagens C-Scan como uma ferramenta auxiliar na detecção dos defeitos. A técnica de processamento de imagens escolhida foi a limiarização multilevel, na qual, para cada amostra, além da imagem original, também é gerada uma nova imagem, denominada de imagem processada. $O$ principal objetivo desta metodologia é realçar os defeitos que simulam a corrosão e eliminar os falsos defeitos (provavelmente estes se referem a variações na espessura do revestimento).

Matematicamente, a limiarização multilevel $[9,10]$ gera como resultado uma imagem processada, $g(x)$, não binarizada, através da alteração da escala de cinza da imagem original, $f(x)$. Este procedimento é baseado na escolha de um limiar primário $(t)$, que se encontra numa região próximo da borda do defeito e, os valores do limiar secundário serão iguais ao valor do elemento inicial da imagem, objetivando eliminar as regiões de falsos defeitos na imagem original e permitindo a diferenciação após o processamento. Dependendo da configuração do sensor, os sinais provenientes dos defeitos podem apresentar valores de amplitude negativos ou positivos, conforme poderá ser observado nas imagens C-Scan do próximo item [3,6]. A metodologia utilizada para eliminar a presença de falsos defeitos e também melhorar a detectabilidade neste trabalho foi baseada no sinal de amplitude apresentado pelo defeito: defeitos que apresentam amplitude positiva foram processados de acordo com a equação 1 e defeitos com sinal negativo de acordo com a equação 2.

$$
\begin{array}{cc}
g(x, y)=f(x, y) & \text { se } f(x, y) \geq t \\
0 & \text { se } f(x, y)<t \\
& \\
g(x, y)=f(x, y) & \text { se } f(x, y) \leq t \\
0 & \text { se } f(x, y)>t
\end{array}
$$

O dimensionamento dos defeitos foi realizado tanto na imagem original como também na imagem processada, através do software Matlab. O cálculo foi baseado na média aritmética da diagonal em $\mathrm{x}$ e em y dos defeitos utilizando a ferramenta brush do software. 


\section{RESULTADOS E DISCUSSÃO}

Na Figura 1 são apresentados os mapas C-Scan obtidos pelas duas metodologias de estudo para a amostra A1. Analisando os dois mapas, observa-se que os defeitos de maior diâmetro apresentam valores de amplitude de aproximadamente $2,5 \mathrm{~V}$ enquanto que os defeitos menores apresentam amplitudes aproximadamente de 1,5 $\mathrm{V}$. $\mathrm{Na}$ imagem referente ao C-Scan original, Figura 1(a), é possível observar que as amplitudes negativas estão associadas a variação na espessura do revestimento. Comparando esta imagem com a imagem obtida pela limiarização multilevel, Figura 1(b), a metodologia utilizada de processamento (equação 1) permitiu eliminar o efeito da variação da espessura do revestimento (lift-off) e também melhorar a detectabilidade dos defeitos, uma vez que houve um aumento tanto no contraste quanto na definição das áreas dos defeitos.

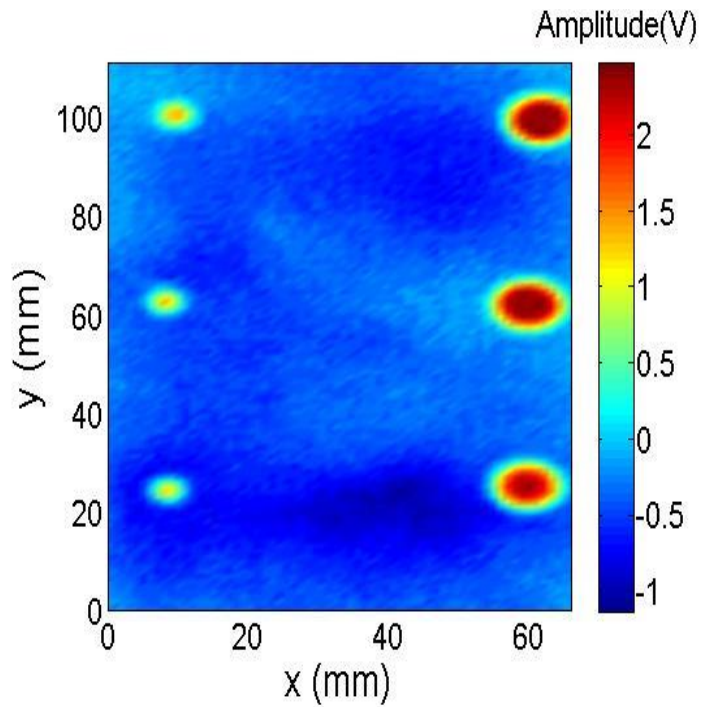

(a)

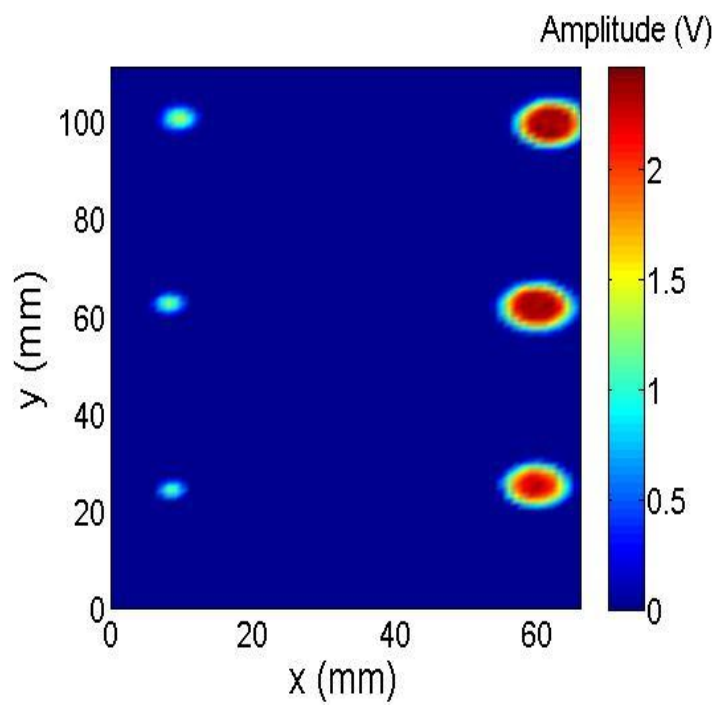

(b)

Figura 1. Resultado obtido da inspeção pela técnica de correntes parasitas para a amostra A1: (a) CScan original e (b) imagem processada.

Na Tabela 3 são apresentados os valores obtidos de dimensionamento para cada um dos defeitos na imagem C-Scan original e na imagem processada. Para os defeitos cujo diâmetro é da ordem de $5 \mathrm{~mm}$, é observado um erro relativo máximo na imagem original de $5 \%$. No entanto, na imagem processada, o erro relativo varia de $24 \%$ a $33 \%$. Para os defeitos de $11 \mathrm{~mm}$ de diâmetro, observa-se um erro relativo que varia de $0 \%$ a $2 \%$ nas duas metodologias (imagem original e imagem processada).

Tabela 3. Dimensionamento dos defeitos da amostra A1

\begin{tabular}{cccccc}
\hline \multicolumn{5}{c}{ Defeito } & \multicolumn{5}{c}{ Amostra A1 } \\
\cline { 2 - 6 } & $\begin{array}{c}\text { Diâmetro } \\
\text { nominal } \\
(\mathrm{mm})\end{array}$ & $\begin{array}{c}\text { Tamanho } \\
\text { medido } \\
(\mathrm{mm})\end{array}$ & $\begin{array}{c}\text { Erro relativo } \\
(\%)\end{array}$ & $\begin{array}{c}\text { Tamanho } \\
\text { medido } \\
(\mathrm{mm})\end{array}$ & $\begin{array}{c}\text { Erro relativo } \\
(\%)\end{array}$ \\
\hline 1 & 5.45 & 5.50 & 1 & 4.00 & 27 \\
\hline 2 & 5.25 & 5.50 & 5 & 3.50 & 33 \\
\hline 3 & 5.25 & 5.25 & 0 & 4.00 & 24 \\
\hline 4 & 10.75 & 10.50 & 2 & 10.50 & 2 \\
\hline 5 & 11.00 & 11.00 & 0 & 11.00 & 0 \\
\hline 6 & 10.84 & 10.75 & 1 & 10.75 & 1 \\
\hline
\end{tabular}


$\mathrm{Na}$ Figura 2 são apresentados os mapas C-Scan obtidos para a amostra A2. Comparando os resultados entre as duas metodologias, observou-se o mesmo comportamento da amostra $\mathrm{A} 1 \mathrm{em}$ relação a amplitude negativa do sinal dos defeitos, Figura 2(a), e a melhoria na detectabilidade dos mesmos pelo processamento de imagem realizado, Figura 2(b).

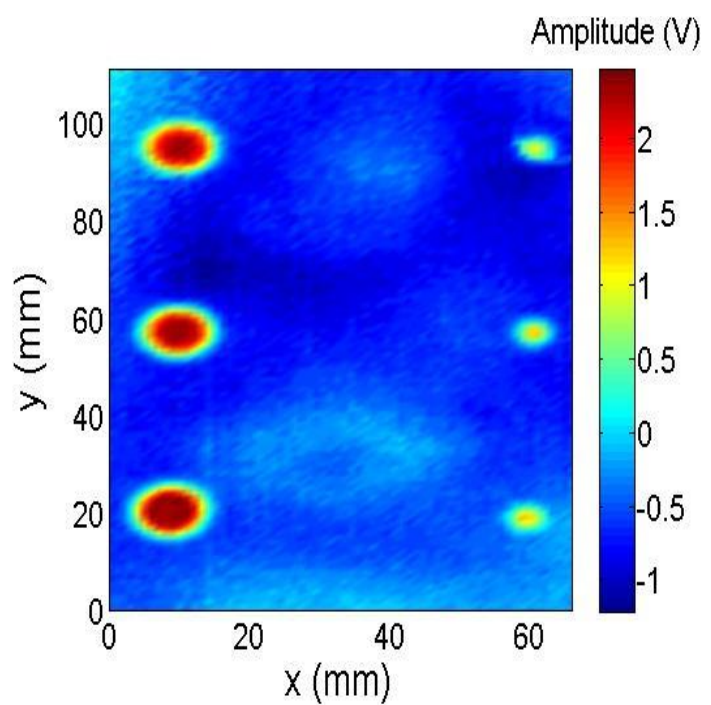

(a)

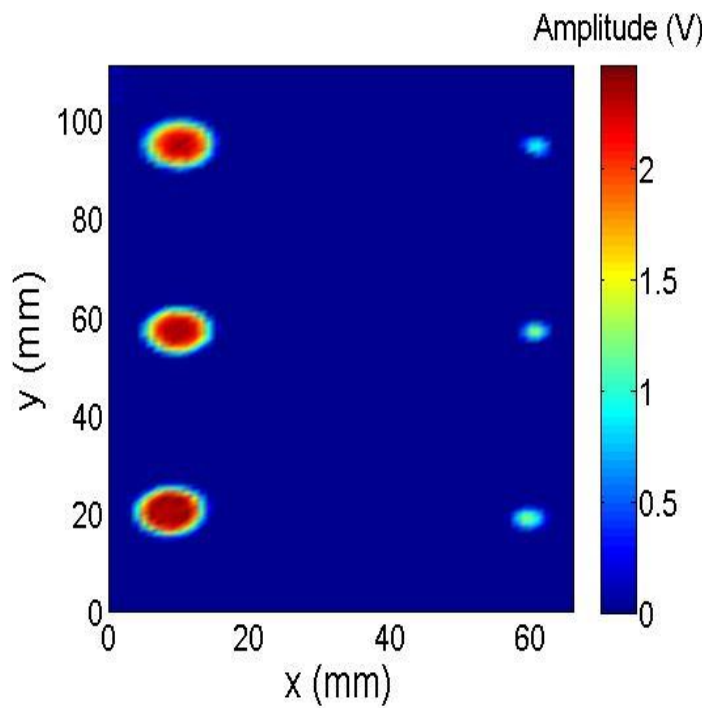

(b)

Figura 2. Resultado obtido da inspeção pela técnica de correntes parasitas para a amostra A2: (a) CScan original e (b) imagem processada.

$\mathrm{Na}$ Tabela 4 são apresentados os valores de dimensionamento obtidos pelas duas metodologias para os defeitos da amostra A2. Na imagem original, Figura 2(a), o dimensionamento dos defeitos apresentou um erro relativo máximo de $7 \%$ e na imagem processada, Figura 2(b), o erro relativo variou entre $4 \%$ a $26 \%$.

Tabela 4. Dimensionamento dos defeitos da amostra A2

\begin{tabular}{cccccc}
\hline Defeito & \multicolumn{5}{c}{ Amostra A2 } \\
\cline { 2 - 6 } & $\begin{array}{c}\text { Diâmetro } \\
\text { nominal } \\
(\mathrm{mm})\end{array}$ & $\begin{array}{c}\text { Tamanhom Original } \\
\text { medido } \\
(\mathrm{mm})\end{array}$ & $\begin{array}{c}\text { Erro relativo } \\
(\%)\end{array}$ & $\begin{array}{c}\text { Tamanho } \\
\text { medido } \\
(\mathrm{mm})\end{array}$ & $\begin{array}{c}\text { Erro relativo } \\
(\%)\end{array}$ \\
\hline 1 & 5.41 & 5.50 & 2 & 4.00 & 26 \\
\hline 2 & 5.36 & 5.50 & 3 & 4.00 & 25 \\
\hline 3 & 5.26 & 5.00 & 5 & 4.00 & 24 \\
\hline 4 & 11.20 & 11.00 & 2 & 10.00 & 11 \\
\hline 5 & 11.20 & 11.00 & 2 & 10.00 & 11 \\
\hline 6 & 11.24 & 10.50 & 7 & 10.74 & 4 \\
\hline
\end{tabular}

$\mathrm{Na}$ Figura 3 são apresentados os mapas C-Scan obtidos para a amostra A3. Na imagem original, Figura 3(a), a técnica se mostrou capaz de detectar todos os três defeitos inseridos na amostra, resultado este que indica que a presença de óxido de ferro nos defeitos não influenciou na capacidade de detecção dos defeitos. Outro comportamento que pode ser observado na imagem original é referente a amplitude negativa dos defeitos de aproximadamente $-2,3 \mathrm{~V}$ e regiões com amplitude positiva que são relacionadas a variações de espessura do revestimento. Após o processamento de imagem (equação 2), Figura 3(b), os defeitos contidos na amostra 
apresentam um maior contraste e definição do que na imagem original devido a eliminação dos falsos defeitos (variação na espessura do revestimento).

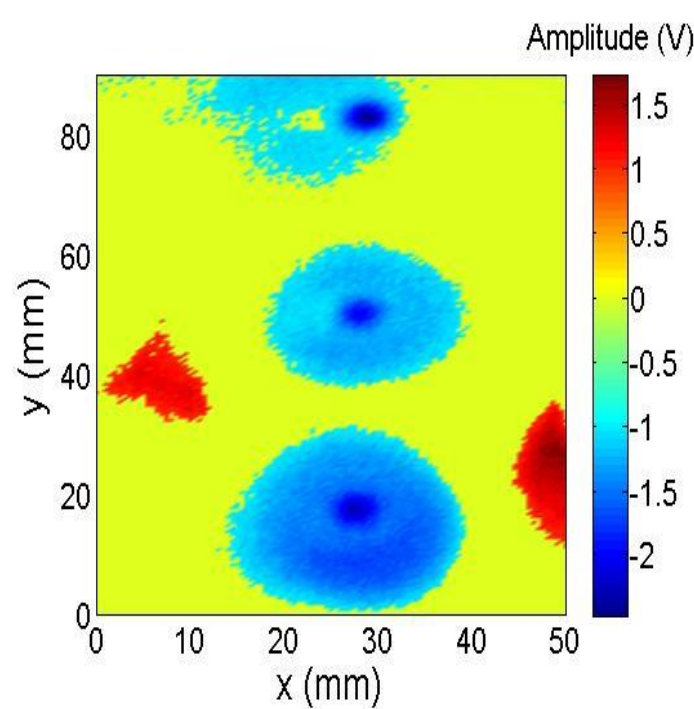

(a)

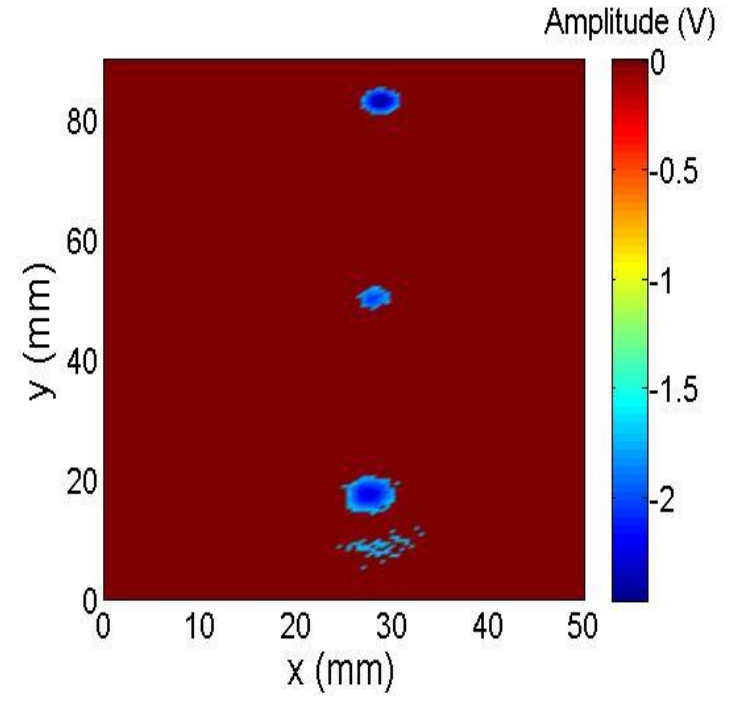

(b)

Figura 3. Resultado obtido da inspeção pela técnica de correntes parasitas para a amostra A3: (a) CScan original e (b) imagem processada.

Os valores de dimensionamento para os defeitos contidos na amostra A3 são apresentados na Tabela 5. O erro relativo obtido para o dimensionamento dos defeitos na imagem original apresentou uma variação de $2 \%$ a $10 \%$. Utilizando a imagem processada, a variação do erro relativo obtido para o dimensionamento foi de $1 \%$ a $28 \%$.

Tabela 4. Dimensionamento dos defeitos da amostra $A 3$

\begin{tabular}{cccccc}
\hline \multicolumn{5}{c}{ Defeito } & \multicolumn{5}{c}{ Amostra A3 } \\
\cline { 2 - 6 } & $\begin{array}{c}\text { Diâmetro } \\
\text { nominal } \\
(\mathrm{mm})\end{array}$ & $\begin{array}{c}\text { Tamanho } \\
\text { medido } \\
(\mathrm{mm})\end{array}$ & $\begin{array}{c}\text { Erro relativo } \\
(\%)\end{array}$ & $\begin{array}{c}\text { Tamanho } \\
\text { medido } \\
(\mathrm{mm})\end{array}$ & $\begin{array}{c}\text { Erro relativo } \\
(\%)\end{array}$ \\
\hline 1 & 5.65 & 5.75 & 2 & 4.50 & 20 \\
\hline 2 & 5.56 & 5.00 & 10 & 4.00 & 28 \\
\hline 3 & 5.53 & 5.00 & 10 & 5.50 & 1 \\
\hline
\end{tabular}

\section{CONCLUSÃo}

Neste trabalho foi avaliada a inspeção por correntes parasitas de estruturas constituídas de um substrato de aço carbono com aplicação de revestimentos compósitos anticorrosivos. A técnica de correntes parasitas se mostrou capaz de detectar defeitos de diferentes geometrias que simulam a perda de espessura no substrato devido a corrosão localizada.

A metodologia de processamento de imagem baseada na limiarização multilevel utilizada na imagem original se tornou uma ferramenta importante para eliminar os sinais de falsos defeitos, ocasionados pela variação da espessura do revestimento (lift-off). Consequentemente, foi possível observar uma melhoria significativa na imagem processada em relação a imagem original, fato este que facilitou a detecção 
dos defeitos. Além disso também observou-se a influência do diâmetro dos defeitos no sinal de amplitude. No entanto, como já esperado, o processamento de imagens gerou um aumento no valor referente ao erro de dimensionamento dos defeitos uma vez que com a aplicação do filtro, alguns dados foram suprimidos na imagem, fato este que influencia diretamente no dimensionamento realizado na imagem processada.

A presença de produto de corrosão preenchendo alguns defeitos, a fim de representar a condição real de corrosão do substrato, não influenciou na detecção dos mesmos. A detecção dos defeitos nesta condição confere a viabilidade de utilização da técnica de correntes parasitas para a inspeção em aplicações industriais.

\section{Agradecimentos}

Os autores agradecem a Petrobras pela assistência técnica e financeira e Capes e CNPq pelo apoio a pesquisa.

\section{REFERÊNCIAS}

1 SØrensen PA, Kiil S, Dam-Johansen K, Weinell CE. Anticorrosive coatings: a review. J. Coatings Technology and Research. 2009; 6(2): 135-176.

2 Udpa SS, Moore PO. Nondestructive Testing Handbook: Eletromagnetic Testing. $3^{\text {rd }}$ ed. American Society for Nondestructive Testing: ASNT; 2005.

3 Angani CS, Park DG, Kim CG, Leela P, Kollu P, Cheong YM. The Pulsed Eddy Current Differential Probe to Detect a Thickness Variation in an Insulated Stainless Steel. J. Nondestructive Evaluation. 2010; 29(4): 248-252.

4 Disque R, Becker M. Multifrequency eddy current (EC) inspection of layered aluminum aircraft structures to detect size hidden corrosion, in: G. Dobmann (Ed.), Eletromagn. Nondestruct. Eval. VII, IOS Press, Saarbrücjen, 2006: pp. 57-61.

5 Pedersen LB, Magnusson KA, Zhengsheng Y. Eddy Current Testing of Thin Layers Using Co-planar Coils. Res. Nondestruct. Eval. 2000; 12(1): 53-64.

6 Park DG, Agnani CS, Kim GD, Cheong YM. Evaluation of Pulsed Eddy Current Response and Detection of the Thickness Variation in the Stainless Steel. IEEE Trans. Magn. 2009; 45(10):3893-3896.

7 Crouzen P, Verweij M, Egink C. Eletromagnetic Profiler for Inspections of Steel trough Corrosion Product. $9^{\text {th }}$ Eur, Conf. ND, 2006: pp. 1-9.

8 Garcia-Martín J, Gómez-Gil J, Vásquez-Sánchez E. Non-Destructive Techniques Based on Eddy Current Testing. Sensors. 2011; 11(3): 2525-2565.

9 Castleman KR. Digital Image Processing. Prentice Hall Press, Upper Saddle River, NJ, USA, 1996.

10 Russ JC. Segmentation and Thresholding. Image Process. Handb., 3th edit., CRC Press LLC, 1998:324-339. 